\title{
THE
}

UNIVERSITY

University of Rhode Island

OF RHODE ISLAND

DigitalCommons@URI

6-15-1990

\section{Cs-Induced Surface State on GaAs(110)}

T. Maeda Wong

N. J. DiNardo

David R. Heskett

University of Rhode Island, dheskett@uri.edu

E. W. Plummer

Follow this and additional works at: https://digitalcommons.uri.edu/phys_facpubs

Terms of Use

All rights reserved under copyright.

\section{Citation/Publisher Attribution}

Maeda Wong, T., DiNardo, N. J., Heskett, D., \& Plummer, E. W. (1990). Cs-induced surface state on GaAs(110). Physical Review B, 41(17), 12342-12345. doi: 10.1103/PhysRevB.41.12342

Available at: http://dx.doi.org/10.1103/PhysRevB.41.12342

This Article is brought to you for free and open access by the Physics at DigitalCommons@URI. It has been accepted for inclusion in Physics Faculty Publications by an authorized administrator of DigitalCommons@URI. For more information, please contact digitalcommons-group@uri.edu. 


\title{
Cs-induced surface state on GaAs(110)
}

\author{
T. Maeda Wong \\ Department of Materials Science and Engineering, University of Pennsylvania, Philadelphia, Pennsylvania 19104-6272 \\ N. J. DiNardo \\ Department of Physics and Atmospheric Science, Drexel University, Philadelphia, Pennsylvania 19104 \\ D. Heskett \\ Department of Physics, University of Rhode Island, Kingston, Rhode Island 02881-0817 \\ E. W. Plummer \\ Department of Physics, University of Pennsylvania, Philadelphia, Pennsylvania 19104-6396
}

(Received 9 August 1989; revised manuscript received 26 March 1990)

Cesium adsorption on GaAs(110) has been studied by angle-resolved photoemission spectroscopy at room temperature in the submonolayer-coverage regime. We report the observation of a $\mathrm{Cs}$-induced surface state in the vicinity of the surface-Brillouin-zone edge. The possible origin of this state is discussed in relation to recent structural observations. The onset of the Cs-induced surface state can be correlated with the appearance of a second Cs $5 p$ core-level emission feature at $\sim 0.2$ monolayer Cs coverage.

A complete understanding of a metal-semiconductor interface requires the correlation of its electronic and geometric properties at all stages of development. These properties are closely related to the physics of Schottkybarrier formation. ' In the initial stages of interfacial metallization, the semiconductor-to-metal transition depends on the density of the metal adlayer and on the electronic structure of the interface. Recently, attention has focused on the adsorption of alkali metals on semiconductor surfaces, particularly $\mathrm{Cs} / \mathrm{GaAs}(110),^{2-5}$ as model systems for the study of interfacial metallization. The clean $\mathrm{GaAs}(110)$ surface exhibits a structural relaxation without reconstruction ${ }^{6}$ and is free of electronic states in the bulk band gap. At submonolayer coverages, scanning tunneling microscopy (STM) has imaged Cs chains along the [110] direction. ${ }^{7}$ The density of the adsorbed alkalimetal layer is an important aspect for the analysis of the interfacial electronic properties. For Cs coverages up to saturation at room temperature, we have determined that the interface is nonmetallic ${ }^{2}$ and that the saturation density is $(4.0 \pm 0.1) \times 10^{14} \mathrm{Cs}$ atoms $/ \mathrm{cm}^{2}$, or 0.9 monolayer (ML), ${ }^{8}$ as determined with medium-energy ion scattering (MEIS). ${ }^{9}$ This Cs density is approximately the surface density of (metallic) Cs(110), which is near the Mott limit for solid Cs, i.e., the density for a three-dimensional (3D) insulator $\rightarrow$ metal transition. ${ }^{10}$ Interfacial metallization occurs upon the development of a second Cs layer formed at low temperature. Magnusson and Riehl have recently studied the midgap electronic structure for this system using angle-integrated photoemission and inverse photoemission spectroscopies. 11

The objective of this paper is to correlate electronic structure at submonolayer Cs coverages with known Cs surface structures ${ }^{7}$ using angle-resolved photoemission spectroscopy. We report the observation of a Cs-induced surface state for room-temperature adsorption at cover- ages, $\theta$, in the range $0.2<\theta<0.5 \mathrm{ML}$, where $\mathrm{STM}$ studies $^{7}$ indicate that three-row Cs chain structures predominate. The state is observed in the band gap near the boundary of surface Brillouin zone (SBZ). In addition, Cs $5 p$ core-level spectra taken in this coverage range distinctly exhibit two components, and the onset of the lower binding-energy component is correlated with the appearance of the Cs-induced surface state.

The experiments were performed at the National Synchrotron Light Source at Brookhaven National Laboratory (Upton, NY). Details of the experimental apparatus have been reported previously. ${ }^{2}$ Overall energy resolution was $-150-200 \mathrm{meV}$ for a photon energy, $h v$, of $30 \mathrm{eV}$. The energy position of the Fermi level was established by photoemission from metallic Cs multilayers grown at low temperature and from the metal sample holder. The $n$ type GaAs (110) wafer (Si-doped, $n_{\mathrm{Si}} \sim 10^{18} \mathrm{~cm}^{-3}$ ) was prepared by cycles of sputtering and annealing. Our angle-resolved photoemission spectra are in excellent agreement with those of cleaved surfaces. ${ }^{12}$ Cs was evaporated from a shuttered SAES Getters/USA, Inc. getter source. Cs $5 p$ photoemission intensities were normalized to absolute coverage measurements obtained by MEIS (Ref. 9) to establish calibrations of exposure and work function change versus absolute coverage.

Angle-resolved photoemission spectra for sequential depositions of $\mathrm{Cs}$ onto the clean $\mathrm{GaAs}(110)$ surface at room temperature are presented in Fig. 1. These spectra were acquired with $25-\mathrm{eV}$ photons incident at $45^{\circ}$ from the surface normal and with $\mathbf{A}_{\|}$directed in the $\bar{\Gamma} \bar{X}$ direction. In order to correct for band bending as measured by shifts in the $\mathrm{Ga}(3 d)$ core level, the valence-band maximum (VBM) is used as the energy reference, and the position of the Fermi level for each spectrum is denoted by a tic mark. For this series of spectra, the analyzer was positioned between points $\bar{X}$ and $\bar{M}$ in the SBZ (inset of Fig. 


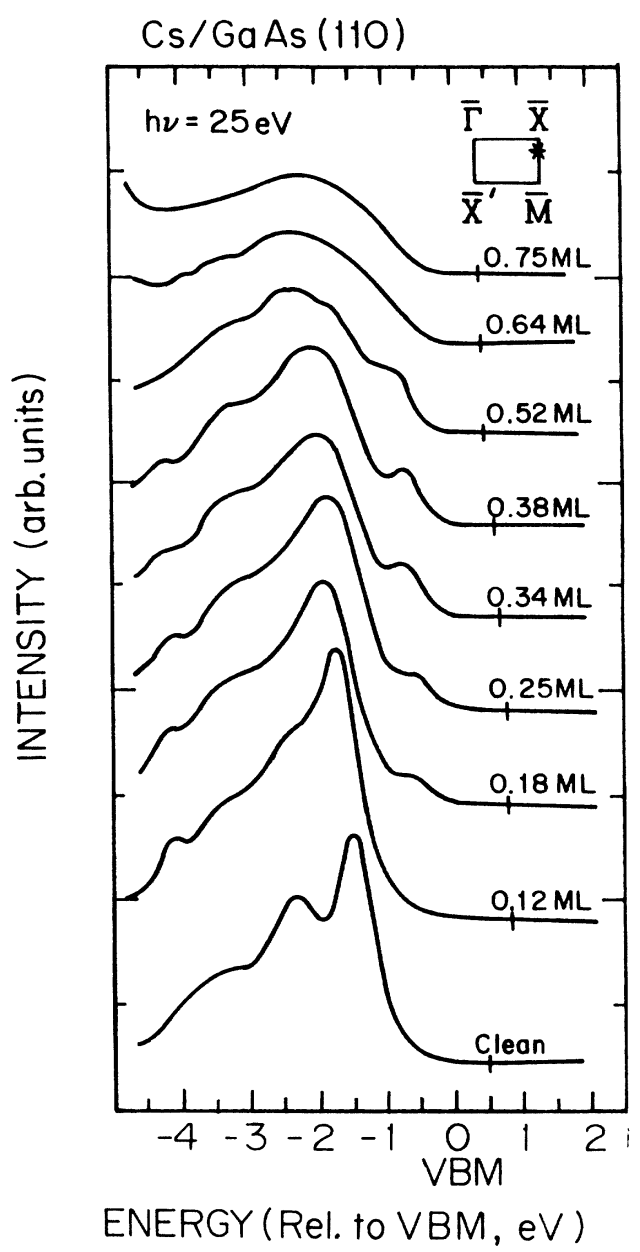

FIG. 1. Angle-resolved photoemission spectra for various Cs coverages obtained with $h v=25 \mathrm{eV}$ and $\mathbf{k}_{\|}$near point $\bar{X}(*)$. The spectra have been corrected for band bending and the binding energies are referenced to the VBM. Tic marks show the position of the $E_{F}$ for each coverage. (Note: $1 \mathrm{ML} \equiv 4.43 \times 10^{14}$ $\mathrm{cm}^{-2}$.)

1) where the Cs-induced valence feature was best observed. Several spectral changes are observed as the Cs coverage is increased. First, attenuation of the As lonepair surface resonance at $\sim-1.45 \mathrm{eV}$ is observed. Second, at $\sim 0.18-M L$ Cs coverage, a new peak appears above the projected valence band (VB) at $\sim-0.45 \mathrm{eV}$. This feature reaches maximum intensity at $\sim 0.38-\mathrm{ML}$ coverage and attenuates beyond $\sim 0.5-\mathrm{ML}$ Cs coverage. After it appears, the feature shifts downwards by $\sim 0.3$ $\mathrm{eV}$ before it is extinguished at higher Cs coverages.

The dispersion of the Cs-induced feature along the $\bar{\Gamma} \bar{X}$ and $\bar{\Gamma} \bar{X}^{\prime}$ directions measured at a Cs coverage of $\sim 0.34$ ML is shown in Fig. 2. The cross-hatched region shows the projected bulk band structure. The dashed lines show the measured surface resonances of clean GaAs(110) which agree with calculations ${ }^{13}$ as well as with previous measurements. ${ }^{12}$ The Cs-induced surface state is observed about halfway out from $\bar{\Gamma}$ along the $\bar{\Gamma} \bar{X}$ and $\bar{\Gamma} \bar{X}^{\prime}$ directions where the projected VB falls below the energy of this feature. We will discuss the dispersion behavior below.

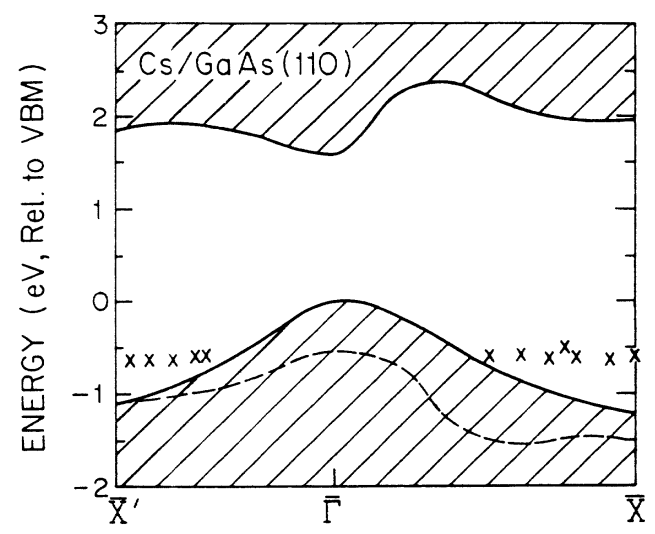

FIG. 2. Measured surface band dispersion for As lone-pair surface resonance on clean $\mathrm{GaAs}(110)$ (dashed line) and for the Cs-induced surface state at $\sim 0.34-M L$ Cs coverage. The cross-hatched region denotes projected bulk $\mathrm{GaAs}$ band structure.

To relate the geometric structure and the electronic properties, we compare our valence spectra with structures reported by STM (Ref. 7) at corresponding coverages. At lowest average Cs coverages, isolated zig-zag double-row chains comprised of two, doubly periodic Cs rows along the [1ㅣㅣㄹ direction laterally displaced by one lattice constant were observed. The analysis of First et $a l^{7}$ indicated that the images represent $\mathrm{Cs}$ atoms existing in equivalent quasithreefold sites associated with one $\mathrm{Ga}$ and two As atoms. Above $\sim 0.12 \mathrm{ML}$, individual zig-zag double-row chains were altered by the addition of "tertiary" Cs rows. The Cs atoms of the tertiary row reside in a local site different from the Cs atoms in the double-row chains with which they are associated. Interactions between adjacent three-row chains appear to be weak since there is little tendency for such structures to coalesce at low coverages. The chains are expected to coalesce at a local coverage of $\boldsymbol{\theta}=0.5$. Then, based on the large size of atomic $\mathrm{Cs}$ and on absolute coverage measurements, ${ }^{9}$ continued adsorption represents a compressional phase of the Cs layer. This coincides with the gradual attenuation of the Cs-induced feature and a reduction of the Cs uptake rate. $^{3}$ Since the $C s$-induced valence state is most prominent for the coverages $\theta \sim 0.2-0.4 M L$, we associate this feature with the presence of three-row Cs structures. We assume that defect sites, if any, would be occupied initially; therefore, a defect-related origin for the Cs-induced state is unlikely since it appears at intermediate Cs coverages. In addition, we note that, if scanning tunneling spectroscopy had been performed, it is unlikely that the Cs-induced surface state could have been observed due to the inherent lack of wave vector selectivity by that technique.

We consider the origin of the observed Cs-induced valence state. The state may originate from a shift in the binding energy of the As lone-pair orbital due to a Csinduced modification of the surface structural relaxation in the vicinity of adsorbed Cs atoms. Calculations indicate that the clean surface relaxation forming $s p^{2}$-like hybridization lowers the energy of the As lone-pair band causing it to mix with the bulk bands throughout the 
SBZ. ${ }^{14}$ Calculations for the clean surface relaxation predict a shift of the As-derived surface band to higher binding energy. ${ }^{14}$ The (local) modification of the surface relaxation by adsorbed $\mathrm{Cs}$ for the three-row structure could shift the As-derived surface band back up towards the bulk band gap. There is evidence that the potential barrier between the relaxed and unrelaxed structures is small. ${ }^{15}$

The negligible dispersion of the Cs-induced surface state would be consistent with an explanation of a local structural change. The dispersion of the As lone-pair band for the clean surface between points $\bar{\Gamma}$ and $\bar{X}$ and between points $\bar{\Gamma}$ and $\bar{X}^{\prime}$ (in regions where it does not mix with the bulk bands) is only $\sim 0.3 \mathrm{eV}$. Upon the formation of Cs chains, the periodicity in the [1 10$]$ direction doubles and this would further reduce the expected dispersion of a band related to the As lone-pair orbitals. Further evidence relating this state to the As lone-pair band can be derived from photon energy-dependent measurements. ${ }^{3}$ The intensity of the Cs-induced state monotonically decreases with increasing photon energy (15 $\mathrm{eV}<h v<35 \mathrm{eV}$ ) similar to the behavior of the As surface resonance of clean $\mathrm{GaAs}(110){ }^{3}$

An alternate explanation for the Cs-induced state is to consider it as being derived from the Cs $6 s$ level involved in the bonding interaction between the adsorbate and the substrate surface. In this case, it is necessary to invoke some sort of Cs pairing, or dimerization, in order to account for the nonmetallic interface found by $\mathrm{us}^{2,3}$ and by Magnusson and Riehl ${ }^{11}$ at these coverages.

The specific Cs structures present in the development of the Cs layer should produce Cs core-level spectra that exhibit separate spectral components for each Cs localbonding environment. A series of Cs $5 p$ photoelectron spectra (normal emission, $h v=31.5 \mathrm{eV}$ ) for sequential Cs depositions is presented in Fig. 3. It should be noted that these spectra and the valence spectra of Fig. 1 were obtained in different experimental runs, but our calibration of coverage versus exposure produces spectral trends which are consistent and reproducible. In qualitative agreement with previous work, ${ }^{4}$ we observe spin-orbit split pairs $\left(5 p_{1 / 2}, 5 p_{3 / 2}\right)$ which are comprised of two components at lower Cs coverages. The observed binding energies are in agreement with other studies for Cs adsorbed on $\mathrm{Al}(111)$ (Ref. 16) and for Cs metal. ${ }^{17}$ The deconvoluted spectra show that, for $\theta \leq 0.4 \mathrm{ML}$, binding energies of the Cs $5 p_{3 / 2}$ components are observed at 11.7 and 11.1 eV below the VBM (with similar splitting observed for the $5 p_{1 / 2}$ components). ${ }^{3}$ At coverages above $\sim 0.45 \mathrm{ML}$, the Cs $5 p$ components begin to coalesce into a broader structure, which may include other Cs $5 p$ contributions due to the coexistence of the compressed structural phase. The bonding state related to the high binding energy Cs $5 p_{3 / 2}$ component (at $-11.7 \mathrm{eV}$ ) dominates at lowest Cs cover-

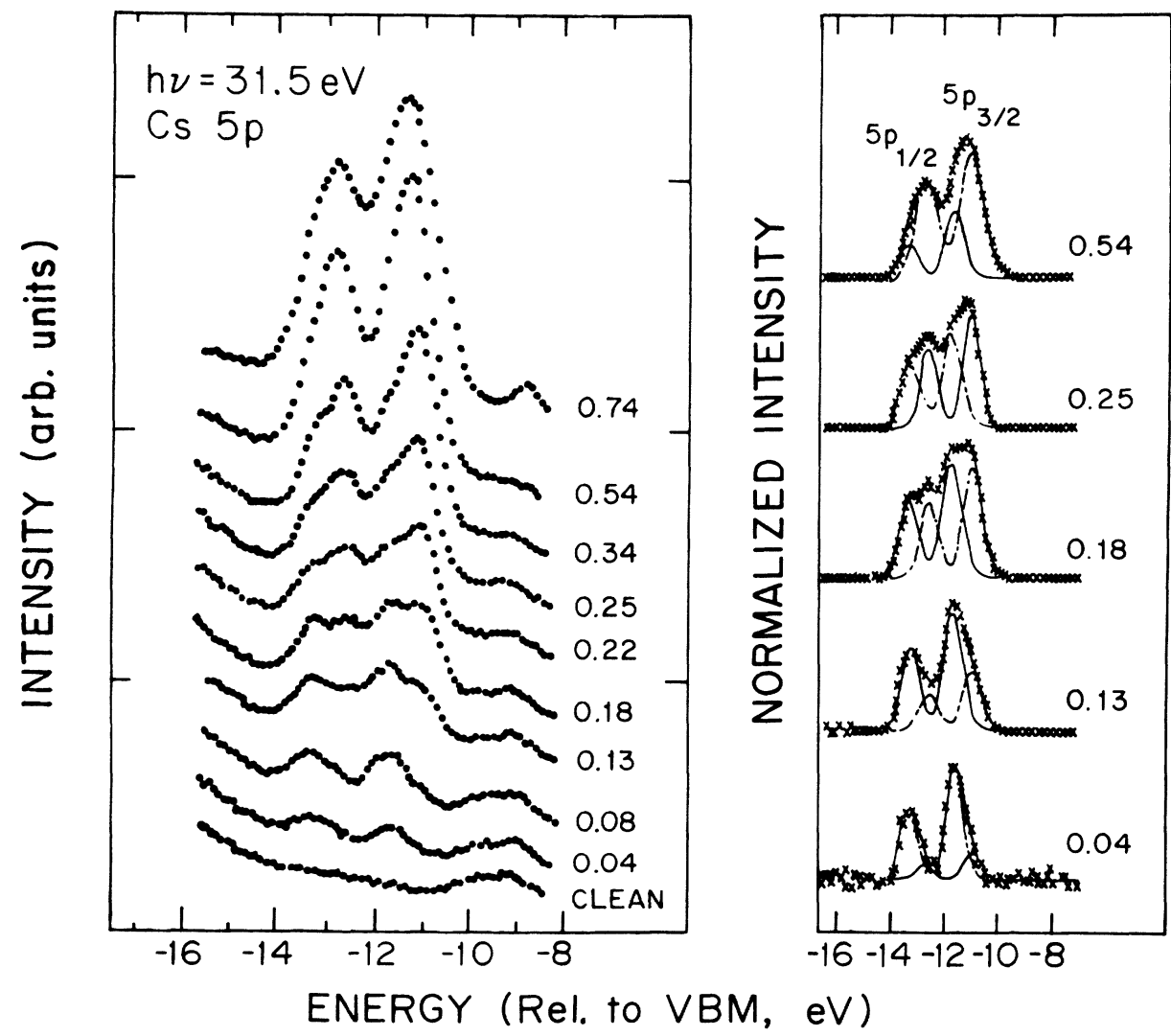

FIG. 3. Left-hand side: Photoemission spectra of Cs $5 p$ core level region with $h v=31.5 \mathrm{eV}$. The spectra have been corrected for band bending and the binding energies are referenced to the VBM. This series of spectra shows the development of a pair of spinorbit split Cs $5 p$ core level components with increasing Cs coverage. Right-hand side: Deconvoluted Cs $5 p$ spectra for selected coverages illustrating the development of two components as a function of coverage. 
ages $(\Theta<0.1 \mathrm{ML})$. The lower binding-energy component appears around the onset coverage for the Cs-induced surface state and its growth predominates up to coverages where the compressional phase begins to form. At that coverage, other component(s) prevent unambiguous spectral deconvolution. Presently, the growth of individual Cs $5 p$ components cannot be correlated with the density of $\mathrm{Cs}$ atoms in specific sites or bonding environments, but we believe the coincidence of the onset between core and valence spectra is significant.

To further support a structural origin of the Cs-induced surface state, its behavior has also been studied as a function of annealing. After room-temperature Cs exposure, annealing to $T \sim 200^{\circ} \mathrm{C}$ caused partial attenuation of the state without Cs desorption (based on the Cs $5 p$ intensity). The Cs $5 p$ spectrum of this annealed interface is similar to that of the room-temperature saturation Cs phase. These observations suggest that the Cs-induced surface state is related to a metastable structural phase formed at room temperature which is irreversibly altered after annealing.
In conclusion, we have observed the development of an occupied Cs-induced surface state in the GaAs band gap using angle-resolved photoemission spectroscopy. We have correlated the appearance of this state with structural phases observed in a previous STM study. ${ }^{7}$ Theoretical calculations and future experiments such as near-edge $x$ ray-absorption fine structure, $x$-ray photoelectron diffraction, or low-energy electron diffraction may further correlate local geometric structure with the electronic properties of the Cs-induced surface state and with the behavior of the Cs $5 p$ core-level components.

This work was supported by U. S. Office of Naval Research Contract No. N00014-86-0304. The synchrotron radiation beam line is operated and maintained by the University of Pennsylvania Materials Research Laboratory (Division of Materials Research, National Science Foundation Grant No. 88-19885). N.J.D. also acknowledges support from Research Corporation. We thank D. M. Zehner for providing the GaAs(110) wafers.
${ }^{1}$ L. J. Brillson, Surf. Sci. Rep. 2, 123 (1982).

${ }^{2}$ T. Maeda-Wong, D. Heskett, N. J. DiNardo, and E. W. Plummer, Surf. Sci. Lett. 208, Ll (1989).

${ }^{3}$ T. Maeda-Wong, D. Heskett, N. J. DiNardo, and E. W. Plummer (unpublished).

${ }^{4}$ T. Kendelewicz, P. Soukiassian, M. H. Bakshi, Z. Hurych, I. Lindau, and W. E. Spicer, Phys. Rev. B 38, 7568 (1988); J. Vac. Sci. Technol. A 6, 1569 (1988).

${ }^{5}$ M. Prietsch, M. Domke, C. Laubschat, T. Mandel, C. Xue, and G. Kaindl, Z. Phys. B 74, 21 (1989).

${ }^{6}$ C. B. Duke, C. Mailhiot, A. Paton, D. J. Chadi, and A. Kahn, J. Vac. Sci. Technol. B 3, 1087 (1985); L. Smit, T. E. Derry, and J. F. van der Veen, Surf. Sci. 150, 245 (1985); R. M. Feenstra, Joseph A Stroscio, J. Tersoff, and A. P. Fein, Phys. Rev. Lett. 58, 1192 (1987), and references therein.

${ }^{7}$ P. N. First, R. A. Dragoset, Joseph A Stroscio, R. J. Celotta, and R. M. Feenstra, J. Vac. Sci. Technol. A 7, 2868 (1989).

${ }^{8} 1 \mathrm{ML} \equiv 4.43 \times 10^{14} \mathrm{~cm}^{-2}$, the density of surface unit cells.

${ }^{9}$ D. Heskett, T. Maeda-Wong, A. Smith, W. R. Graham, N. J.
DiNardo, and E. W. Plummer, J. Vac. Sci. Technol. B 7, 915 (1989).

${ }^{10}$ A. Ferraz, N. H. March, and F. Flores, J. Phys. Chem. Solids 45, 627 (1984).

${ }^{11}$ K. O. Magnusson and B. Riehl, Phys. Rev. B 40, 5864 (1989).

${ }^{12}$ A. Huijser, J. van Laar, and T. L. van Rooy, Phys. Lett. 65A, 337 (1978); J. A. Knapp and G. J. Lapeyre, J. Vac. Sci. Technol. 13, 757 (1976); T.-C. Chiang, J. A. Knapp, M. Aono, and D. E. Eastman, Phys. Rev. B 21, 3513 (1980).

${ }^{13}$ S. B. Zhang and Marvin L. Cohen, Surf. Sci. 172, 754 (1986).

${ }^{14}$ C. Mailhiot, C. B. Duke, and Y. C. Chang, Phys. Rev. B 30, 1109 (1984).

${ }^{15}$ U. Harten and J. P. Toennies, Europhys. Lett. 4, 833 (1987); Y. R. Wang and C. B. Duke, Phys. Rev. B 39, 5569 (1989).

${ }^{16}$ A. Hohlfeld, M. Sunjic, and K. Horn, J. Vac. Sci. Technol. A 5, 679 (1987).

${ }^{17}$ G. Ebbinghaus, W. Braun, and A. Simon, Z. Naturforsch. 31b, 1219 (1976). 